\title{
Nothing unusual: bilateral recurrent laryngeal nerves have followed the rule
}

\author{
Sudesh Prabhu ${ }^{1}\left(10 \cdot\right.$ Siddhant Mehra ${ }^{1}$
}

Received: 11 July 2020 / Accepted: 11 September 2020 / Published online: 18 September 2020

○) Springer-Verlag France SAS, part of Springer Nature 2020

Yan et al. [4] have explained the course of bilateral recurrent laryngeal nerves (RLN) in a cadaver. The description of the aortic arch anatomy suggests that it is a right aortic arch (RAA) with an aberrant left subclavian artery with a Kommerall diverticulum and a left sided ductus/ligamentum, with the possibility of a circumflex aorta which is difficult to ascertain as it is a cadaveric study. The anatomy was explained in 1963 by Felson et al. [2] and again in 1970 by Shuford et al. [3] with embryological basis. Embryologically the RAA arises from the 4th right primitive branchial arch vessel and the RLN always courses around the distal primitive 6th arch vessel. The final course of the RLN is decided by the disappearance of the branchial arch vessels during development.

In the discussion Yan et al. [4] have mentioned that in their case, the right RLN turned ventrally under the RAA (6th branchial arch artery; but not the 4th branchial arch artery, namely right subclavian artery), and the left one also turned ventrally under the arch (6th branchial arch artery, but not dorsally). But this explanation is incorrect, as in a RAA the arch is a derivative of the 4th branchial arch artery (not 6th as mentioned) and the right subclavian artery is purely a derivative of the 7 th cervical intersegmental artery (not the 4th branchial arch artery as mentioned).

In the described case the bilateral RLNs have followed the rule. The right sided distal 6th and 5th branchial arch vessels have disappeared and the 4th branchial artery has formed the arch, hence the right RLN has coursed around the aortic arch. And the right subclavian artery being solely derived from the 7th cervical intersegmental artery, the RLN cannot wind around it. Similarly on the left side, the distal 6th branchial arch vessel has given rise to the ductus/

Sudesh Prabhu

sudesh006@gmail.com

1 Department of Pediatric Cardiac Surgery, Narayana Institute of Cardiac Sciences, Bangalore 560099, Karnataka, India ligamentum and as per the rule the left RLN has to course around it.

With the above embryological explanation which dates back to Rathke's arch diagram and Edward's concept of double aortic arch, the explained anatomy should not be considered as unusual course of RLNs [1].

Author contributions Both authors-literature review and manuscript preparation.

Funding None.

\section{Compliance with ethical standards}

Conflict of interest The authors declare that they have no conflict of interest.

\section{References}

1. Edwards JE (1948) Anomalies of the derivatives of the aortic arch system. Med Clin N Am 32:925-949. https://doi.org/10.1016/ s0025-7125(16)35662-0

2. Felson B, Palayew MJ (1963) The two types of right aortic arch. Radiology 81:745-759. https://doi.org/10.1148/81.5.745

3. Shuford WH, Sybers RG, Edwards FK (1970) The three types of right aortic arch. Am J Roentgenol Radium Ther Nucl Med 109:67-74. https://doi.org/10.2214/ajr.109.1.67

4. Yan J, Kanazawa J, Numata N, Hitomi J (2017) The right-sided aortic arch with unusual course of bilateral recurrent laryngeal nerves: a report of rare variations. Surg Radiol Anat 39:223-228. https://doi.org/10.1007/s00276-016-1717-7

Publisher's Note Springer Nature remains neutral with regard to jurisdictional claims in published maps and institutional affiliations. 